\title{
Validity of Selection Breeding for High Ephedrine-Alkaloid Content in E. sinica under Different Environments in Japan
}

\author{
Hajime Hiyama, ${ }^{*, a, b}$ Aya Ozawa, ${ }^{c}$ Bunsho Makino, ${ }^{c}$ Yosuke Yoshioka, ${ }^{d}$ and Ryo Ohsawa ${ }^{*, d}$ \\ ${ }^{a}$ Botanical Raw Materials Production Department, TSUMURA \& CO.; 3586 Yoshiwara, Ami-machi, Inashiki-gun, \\ Ibaraki 300-1192, Japan: ${ }^{b}$ Graduate School of Life and Environmental Sciences, University of Tsukuba; 1-1-1 \\ Tennodai, Tsukuba, Ibaraki 305-8572, Japan: ${ }^{c}$ Botanical Raw Materials Research Laboratories, TSUMURA \& \\ CO.; 3586 Yoshiwara, Ami-machi, Inashiki-gun, Ibaraki 300-1192, Japan: and ${ }^{d}$ Faculty of Life and Environmental \\ Sciences, University of Tsukuba; 1-1-1 Tennodai, Tsukuba, Ibaraki 305-8572, Japan.
}

Received September 23, 2020; accepted November 26, 2020

Dried terrestrial stems of Ephedra sinica are called 'Ephedra herb,' whose pharmacological effects are due mainly to two major ingredients, (-)-ephedrine and (+)-pseudoephedrine (total alkaloids which are defined in Japanese Pharmacopoeia (TA)). Ephedra herb is an important crude drug in Japan. However, $E$. sinica is widely distributed in arid areas of northeastern China and Mongolia. Recently, $E$. sinica has started to be cultivated in Japan. This study aimed to assess the validity of selection breeding on TA content of $E$. sinica in several locations in Japan. In this experiment, we grew approximately 350 seedlings and divided them randomly into seven groups. Nearly fifty plants were cultivated at each of seven locations. In Ibaraki, Yamanashi, and Shizuoka, average TA content of whole samples satisfied the criteria for Ephedra herb defined in Japanese Pharmacopoeia ( $7.0 \mathrm{mg} / \mathrm{g}$ of dry weight (DW)). Plants with high and intermediate TA content at four locations were selected and transplanted to Ibaraki. There were significant differences in TA content between selected plants with high and intermediate TA content before and after transplanting $(p<0.05)$. TA content of high-TA plants was significantly higher than that of control plants cultivated continuously at Ibaraki $(p<0.05)$. These results suggest that the selection on content of ephedrine alkaloids in $E$. sinica under various locations in Japan is valid, and high- TA $E$. sinica plants can be selected at various locations.

Key words Ephedra sinica; total alkaloids defined in Japanese Pharmacopoeia; breeding; selection

\section{INTRODUCTION}

Ephedra sinica Stapf is a dioecious, gymnosperm shrub distributed across arid areas of northern China, and Mongolia. ${ }^{1)}$ Its dried terrestrial stems, known as 'Ephedra herb,' are an important component of many traditional Japanese (Kampo) and Chinese medicinal prescriptions. Ephedra herb has been used traditionally for the treatment of the common cold, headache, bronchial asthma, and nasal inflammation. Japanese Pharmacopoeia states that Ephedra herb for medicinal use must be over $0.7 \%$ in total alkaloids which are defined in Japanese Pharmacopoeia (TA) content $(7.0 \mathrm{mg} / \mathrm{g}$ dry weight (DW)). ${ }^{2)}$

Although Ephedra sinica has been cultivated commercially since the 1980s in China, ${ }^{3)}$ recently, it has also started to be cultivated in Japan. ${ }^{4)}$ However, inclusion of Ephedra herb in Kampo medicine relies on imports from China. Therefore, it would be desirable to cultivate E. sinica in Japan to ensure a stable supply of Ephedra herb for the pharmaceutical industry.

Recently, numerous studies of various factors affecting ephedrine alkaloids have been reported. Some of these studies reported that the content of ephedrine alkaloids in E. sinica is affected by environmental factors. ${ }^{5,6)}$ Additionally, ephedrinealkaloid content can reportedly vary widely with habitat. $5,7,8)$ However, the ephedrine-alkaloid composition ratio seemingly depends on genetic factors but not on environmental factors or growth period. ${ }^{9)}$ Therefore, it is necessary to breed E. sinica for high and stable ephedrine-alkaloid content for cultivation in Japan, as very few researches on breeding for ephedrine alkaloids have been reported. Our previous study demonstrated that it is possible to stabilize the TA content of E. sinica using selection breeding. ${ }^{10)}$ However, that study was performed in a single location, while it is necessary to consider that local environment might affect the selection. Thus, we need to assess whether plants of $E$. sinica with higher TA content can be universally selected under different locations for the establishment of selection breeding in Japan.

In this study, we cultivated E. sinica at seven different locations in Japan to evaluate the validity of selection for TA content. Thus, individuals with high and intermediate content were selected at each location. Then, the selected plants were transplanted to Ibaraki Prefecture, a type of common garden experiment to prove that the effect of selection under different environments is genetic, thereby establishing the validity of the selection beyond potential environmental effects in each different area.

\section{MATERIALS AND METHODS}

Plant Material E. sinica plants were raised from seed for a year in an experimental field in Ibaraki Prefecture in 2012. The seeds used originated in Inner Mongolia from open-pollinated populations. A total of 333 E. sinica plants were randomly selected. The plants used in this study were identified by the author (Hiyama H). Voucher specimens (THS 94868) were deposited in the Herbarium of TSUMURA Botanical Raw Material Research Laboratories, Tsumura \& Co., Ibaraki, Japan.

Experimental Design Three hundred and thirty three seedlings were randomly divided into seven groups for cul- 
Table 1. Environmental Data for the Seven Japanese Locations Evaluated for E. sinica Cultivation

\begin{tabular}{|c|c|c|c|c|c|c|c|}
\hline \multirow{2}{*}{ Factor } & \multicolumn{3}{|c|}{ Hokkaido } & \multirow{2}{*}{ Ibaraki } & \multirow{2}{*}{ Yamanashi } & \multirow{2}{*}{ Shizuoka } & \multirow{2}{*}{ Okinawa } \\
\hline & Eniwa & Toyoura & Taiki & & & & \\
\hline Average temperature $\left({ }^{\circ} \mathrm{C}\right)$ & 7.1 & 7.4 & 5.9 & 14.3 & 11.0 & 16.2 & 22.3 \\
\hline Precipitation (mm) & 948 & 1001 & 1026 & 1341 & 1115 & 2038 & 2500 \\
\hline Day length (h) & 1896 & 1802 & 2193 & 2181 & 2335 & 2325 & 1921 \\
\hline Soil type & Andosol & Andosol & Brown Forest soil & Andosol & Andosol & Lowland soil & Lowland soil \\
\hline Soil texture & Loam & Loam & Loam & Loam & Loam & Sandy loam & Sandy loam \\
\hline Soil pH & 5.6 & 5.5 & 5.6 & 6.5 & 6.9 & 5.8 & 8.1 \\
\hline North latitude & $42^{\circ} 92^{\prime}$ & $42^{\circ} 65^{\prime}$ & $42^{\circ} 53^{\prime}$ & $35^{\circ} 99^{\prime}$ & $35^{\circ} 78^{\prime}$ & $34^{\circ} 64^{\prime}$ & $26^{\circ} 31^{\prime}$ \\
\hline East longitude & $141^{\circ} 56^{\prime}$ & $140^{\circ} 66^{\prime}$ & $143^{\circ} 27^{\prime}$ & $140^{\circ} 19^{\prime}$ & $138^{\circ} 32^{\prime}$ & $138^{\circ} 11^{\prime}$ & $127^{\circ} 95^{\prime}$ \\
\hline
\end{tabular}

tivation at seven locations in Japan. Random selection of 50 individuals within a single seed population is thought to be representative of the variability of the sample. Therefore, approximately 50 plants were cultivated at each of seven Japanese locations selected from north to south based on latitude (Table 1). Seedlings were transplanted at each location at a spacing of 1.0 and $0.3 \mathrm{~m}$ between and within rows, respectively, from March to May, in 2013. As a basal fertilizer, $2000 \mathrm{~g} \cdot \mathrm{m}^{-2}$ of farmyard manure was used. Chemical fertilizer was applied to supply $2,2,2$, and $0.2 \mathrm{~g} \cdot \mathrm{m}^{-2}$ of $\mathrm{N}, \mathrm{P}_{2} \mathrm{O}_{5}, \mathrm{~K}_{2} \mathrm{O}$, and $\mathrm{Mg}$, respectively. Climatic data for all locations were obtained from the records of the Japan Meteorological Agency. ${ }^{11)}$

Kajimura et $a l .{ }^{12)}$ reported that ephedrine-alkaloid content in Ephedra plants is low in the early stages of stem growth and then stabilizes when the growth of the terrestrial stems stops. Therefore, samples were collected after stem growth stopped. Previous research suggested that TA content in $E$. sinica plants is stable in three-year-old plants. ${ }^{10)}$ In this study, one-year-old stems of three-year-old plants were harvested. The harvest date at the seven locations were as follows: July 18 in Shizuoka $(n=50)$, July 23 in Okinawa $(n=50)$, July 28 in Yamanashi $(n=50)$, August 4 , in Ibaraki $(n=44)$, October 22 in Taiki $(n=56)$, October 23 in Eniwa $(n=37)$, and Toyoura $(n=46)$.

We defined high TA content and intermediate TA content as follows: (1) high TA content comprised the top 10\% TA content in plants cultivated at each location; (2) intermediate TA content, comprised 30 to $70 \%$ TA content in plants cultivated at each location.

In all, 32 plants, four with high TA content and four with intermediate TA content, were selected from four locations (Hokkaido Taiki, Ibaraki, Yamanashi, and Shizuoka), respectively. In Hokkaido Eniwa, Hokkaido Toyoura, and Okinawa, plants were not selected because many plants died.

Then, 24 plants selected at Hokkaido Taiki, Yamanashi, and Shizuoka were transplanted to Ibaraki on October 30, 2015, using the same spacing as before. Four plants selected from Ibaraki prefecture have been cultivated continuously since 2013. Chemical fertilizer was applied as described above.

The harvest date of stems in the selected 32 plants and non-selected 36 plants cultivated at Ibaraki was September 12, 2017. A previous study ${ }^{10)}$ reported that the TA content in terrestrial stems of the transplanted plants was low in the first year. Therefore, although stems of the transplanted plants were harvested in 2016, their TA content was not evaluated in 2016.

Harvest and Processing One-year-old stems of E. sinica plants were cut at approximately $5 \mathrm{~cm}$ above the ground. To avoid mixing with stems of the previous year, stems were cut every year. Because stem age affects TA content, we cut the stems each year but harvested only one-year-old stems.

Stems were dried at $35^{\circ} \mathrm{C}$ for three days and then at $50^{\circ} \mathrm{C}$ for six hours in a food dryer (TB-60-F, Taikisangyo Co., Ltd., Okayama, Japan) to determine their TA content and DW. The samples were ground to a powder using a vibrating rod mill (TI-100, CMT Co., Ltd., Japan).

Determination of TA Quantitative analysis of TA content was performed by HPLC on an instrument (Shimadzu Corp., Japan) equipped with an Inertsil ODS-3 column $(5 \mu \mathrm{m}$, $4.6 \times 150 \mathrm{~mm}$; GL Sciences Inc., Japan). (-)-ephedrine (Eph) and $(+)$-pseudoephedrine $(\mathrm{PEph})$ standards were provided by Tsumura \& Co. (Japan). A mixture of water, acetonitrile, and phosphoric acid $(650: 350: 1, \mathrm{v} / \mathrm{v} / \mathrm{v})$ with $0.5 \%$ sodium dodecyl sulfate was used as the mobile phase. The flow rate and column temperature were kept constant at $1.1 \mathrm{~mL} / \mathrm{min}$ and $40^{\circ} \mathrm{C}$, respectively. The powdered samples $(0.25 \mathrm{~g})$ were extracted with $50 \%$ aqueous methanol $(20 \mathrm{~mL})$ using a reciprocal shaker for $30 \mathrm{~min}$, followed by centrifugation (3000 rpm, $10 \mathrm{~min}$ ). After collection of the supernatant, the residue was re-extracted in the same manner. The combined supernatants were prepared by adding $50 \%$ aqueous methanol to a total volume of $50 \mathrm{~mL} ; 10 \mu \mathrm{L}$ aliquots were injected into the HPLC apparatus.

Statistical Analysis One-way ANOVA was performed to test location effects on TA content or DW and Tukey-Kramer tests were used for multiple mean comparison. Student's $t$ tests were conducted to test for significant differences in TA content between high- and intermediate-TA selected plants. In turn, Dunnett's test was performed to test for significant differences in TA content between selected high-TA and control plants. Finally, Spearman's rank correlation analysis was used to assess the stability of TA content of selected plants. These analyses were performed using the statistical software R (version 3.5.0) https://www.r-project.org/.

\section{RESULTS}

Significant $(p<0.05)$ differences in mean TA content and among the seven locations were detected (Fig. 1). The variation in TA content was large among locations, in three of which (i.e., Ibaraki, Yamanashi, and Shizuoka), mean TA content satisfied the criteria for Ephedra herb defined in Japanese Pharmacopoeia ${ }^{2)}(\geq 7.0 \mathrm{mg} / \mathrm{g} \mathrm{DW})$ and was significantly $(p<0.05)$ higher than those at the other locations. In contrast, mean TA content at three locations (Hokkaido Toyoura, Hokkaido Taiki, and Okinawa) were significantly $(p<0.05)$ lower. 

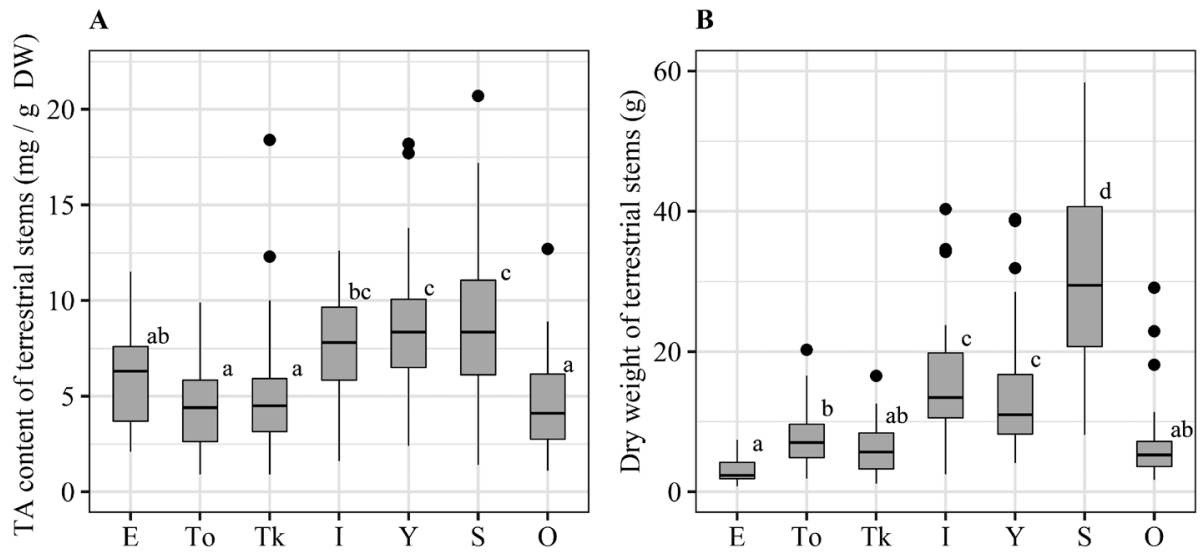

Fig. 1. TA Content and Dry Weight of the Terrestrial Stems of E. sinica Plants Cultivated at Seven Different Locations in Japan, in 2014

A; TA content of plants cultivated at seven locations B; dry weight of plants cultivated at seven locations. Different lowercase letters on the bars represent significant differences $(p<0.05$, Tukey-Kramer multiple-comparison test). E, To, Tk, I, Y, S, and O indicate Hokkaido Eniwa $(n=37)$, Hokkaido Toyoura $(n=46)$, Hokkaido Taiki $(n=56)$, Ibaraki $(n=44)$, Yamanashi $(n=50)$, Shizuoka $(n=50)$, and Okinawa $(n=50)$, respectively.
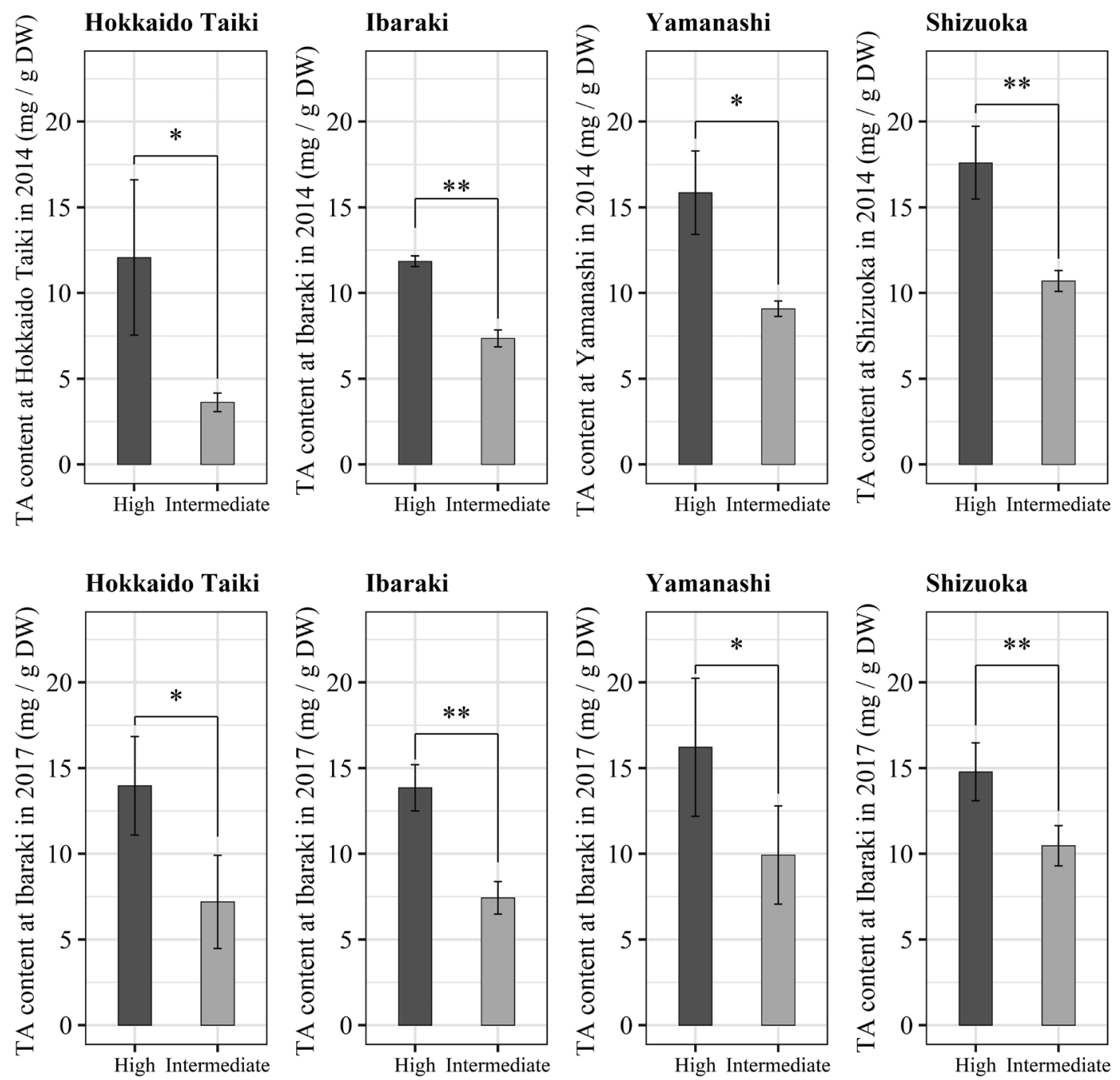

Fig. 2. Relationships between the Selected Plant Groups with High and Intermediate-TA Content before (at Original Locations, 2014) and after Transplant to Ibaraki (2017)

Hokkaido Taiki, Ibaraki, Yamanashi, and Shizuoka indicate each original location of selected plants. High; high-TA plants $(n=4)$. Intermediate; intermediate-TA plants $(n=4)$. The Student's $t$ test was used to evaluate significant differences of TA content between selected high- and intermediate-TA plants. *, **; probability value for testing significance, $<0.05$ and $<0.01$, respectively. 


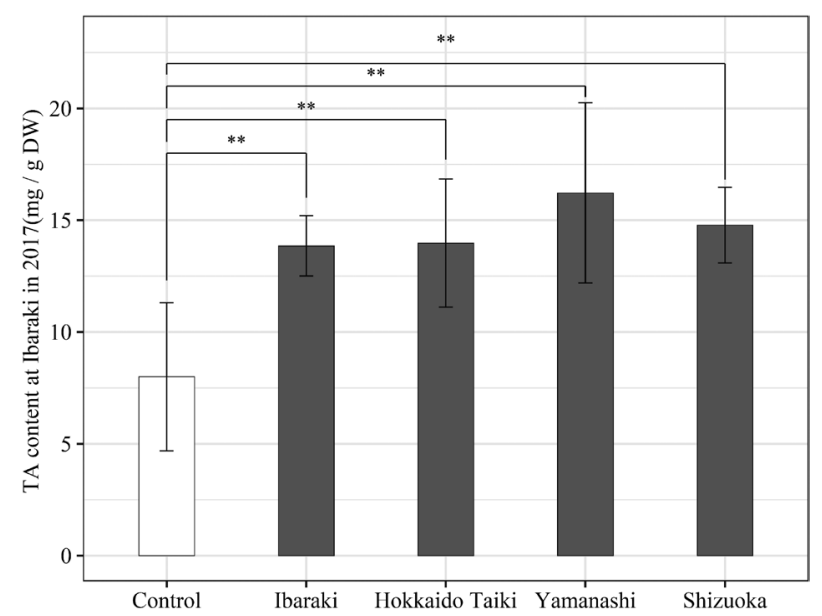

Fig. 3. TA Content in Selected High-TA and Control Plants Cultivated at Ibaraki in 2017

Ibaraki $(n=4)$, Hokkaido Taiki $(n=4)$, Yamanashi $(n=4)$, and Shizuoka $(n=4)$ indicate original location of selected plants. Control $(n=44)$ indicates plants which have been cultivated at Ibaraki since 2013. Dunnett's test was performed to test significant differences in TA content between selected and control plants. ** Probability value for testing significance $<0.01$.

The variation in DW was large among locations, whereby significant $(p<0.05)$ differences in mean stem DW among the seven locations were detected (Fig. 1). In particular, mean DW in Shizuoka was significantly higher than at any other location $(p<0.05)$. In contrast, mean DW at four locations (Hokkaido Eniwa, Hokkaido Toyoura, Hokkaido Taiki, and Okinawa) was significantly $(p<0.05)$ lower.

The TA content of plants selected as high-TA plants cultivated at each location varied from 8.6 to $20.7 \mathrm{mg} / \mathrm{g}$ DW (Supplementary Fig.). Similarly, the TA content of selected high-TA plants transplanted and cultivated at Ibaraki varied from 11.7 to $21.4 \mathrm{mg} / \mathrm{g}$ DW. Therefore, the TA content of all selected high-TA plants fully satisfied the criteria for Ephedra herb defined in Japanese Pharmacopoeia ${ }^{2}$ (i.e., $\geq 7.0 \mathrm{mg} / \mathrm{g}$ DW). Spearman's rank correlation coefficients of TA content before and after transplant were 1.00, 0.76, 0.81, and 0.79 at Hokkaido Taiki, Ibaraki, Yamanashi, and Shizuoka, respectively, and were all significant.

There were significant differences in mean TA content between selected high- and intermediate-TA plants $(p<0.05)$ at each location in 2014 (Fig. 2). Similarly, there were significant differences in mean TA content between selected high-TA and intermediate-TA plants after transplant in $2017(p<0.05)$. Further, there were significant differences between mean TA content of control plants continuously cultivated at Ibaraki from 2013, and mean TA content of selected high-TA plants derived from Ibaraki $(p<0.05)$ (Fig. 3). Moreover, there were significant differences between mean TA content of control plants continuously cultivated at Ibaraki from 2013, and mean TA content of selected high-TA plants transplanted to Ibaraki from the other locations $(p<0.05)$. Lastly, there were no significant differences among mean TA content of selected highTA plants after transplant.

\section{DISCUSSION}

Our experimental cultivation revealed that TA content of E. sinica cultivated at three locations (Ibaraki, Yamanashi, and Shizuoka) in Japan satisfied the criteria for Ephedra herb
( $\geq 7.0 \mathrm{mg} / \mathrm{g}$ DW) defined in Japanese Pharmacopoeia ${ }^{2)}$ (Fig. 1). These three locations, differed from its natural habitat (Table 1), are therefore suitable for cultivation of E. sinica in Japan in terms of TA content.

Our results suggest that the DW of E. sinica was also influenced by local environment. In Shizuoka, mean DW was significantly higher than at the other locations (Table 1, Fig. 1). Therefore, in terms of both TA content and DW, Shizuoka was the most suitable location for cultivation of E. sinica in this study.

Although the TA content of the selected plants was influenced by local environment and varied before and after transplant, the rank correlation coefficients for TA content were significantly high (Supplementary Fig.), meaning that TA content of the selected plants was stable regardless of transplant or location. Moreover, there were significant $(p<0.05)$ differences in mean TA content between high- and intermediate-TA plants before and after transplant (Fig. 2). These results suggest that selection for high TA content was valid regardless of location or transplant. Additionally, there were significant $(p<0.05)$ differences between mean TA content of control and selected plants (Fig. 3). Therefore, it is possible to select E. sinica for high TA content within populations derived from natural variation under the various locations evaluated in Japan. Additionally, although TA content is influenced by local environment, the data showed that the selection of $E$. sinica for high TA content at various locations in Japan is valid. These findings support our previous study ${ }^{10)}$ and suggest that selection breeding of $E$. sinica for high TA content can be performed at various locations. Therefore, high-TA Ephedra herb can be produced stably in Japan, outside its natural habitat, through selection breeding.

It is unclear how TA content in E. sinica is influenced by the environment and what the specific environmental factors are, which determine TA content. However, measuring genotype by environment $(\mathrm{G} \times \mathrm{E})$ interaction is crucial to analyze this issue. In general, $\mathrm{G} \times \mathrm{E}$ interactions are evaluated by cultivating a large number of lines at various locations. In this study, we could not evaluate the $\mathrm{G} \times \mathrm{E}$ interaction effect on TA content or DW in E. sinica because it was difficult to grow a large number of the various clonal lines of $E$. sinica. In the future, many clones of the useful 16 selected plants will be propagated as lines for the evaluation of $\mathrm{G} \times \mathrm{E}$ interactions.

In conclusion, here we identified several Japanese locations that proved suitable for cultivation of E. sinica, as that the resulting TA content satisfied the criteria for Ephedra herb defined in Japanese Pharmacopoeia, and the selection of $E$. sinica for TA content under various locations was validated. Therefore, Ephedra herb high in TA content can be obtained by selection breeding at various locations and can potentially be produced consistently, even in Japan.

Acknowledgments The authors gratefully acknowledge H. Kumazawa, H. Akahori, S. Sasaki, T. Kurosawa, and A. Uetake, M. Sakai for sampling and sample processing.

Conflict of Interest This study was funded by Tsumura \& Co. (https://www.tsumura.co.jp/english/). The funder provided support in the form of a salary for Hajime Hiyama, Aya Ozawa, and Bunsho Makino. Yosuke Yoshioka and Ryo Ohsawa are supervisors at the University of Tsukuba, to 
which Hajime Hiyama belongs, and there was no funding from Tsumura \& Co.

Supplementary Materials The online version of this article contains supplementary materials.

\section{REFERENCES}

1) Price RA. Systematics of the Gnetales: a review of morphological and molecular evidence. Int. J. Plant Sci., 157 (Suppl. 6), S40-S49 (1996).

2) Society of Japanese Pharmacopoeia. The Japanese Pharmacopoeia, 17th ed., Tokyo (2016).

3) Mikage M, Kakiuchi N. The recent situation of the resources of Chinese crude drug Ma-huang, Ephedrae Herba. J. Tard. Med., 22 (Suppl.1), 61-69 (2005).

4) Hokkoku Shimbun. "Kakkonto genryo shukka ni medo Togi de ryosan seiko, 100 kiro mitooshi" (Large-scale cultivation of Mao, one of the raw materials of Kampo medicine, Kakkon to, was successful in Togi (Ishikawa Pref). It will be put prospects on shipment in $100 \mathrm{Kg}$ scale.), Hokkoku Shimbun 04 January 2020.

5) Wang LL, Kakiuchi N, Mikage M. Studies of Ephedra plants in Asia. Part 6: geographical changes of anatomical features and alkaloids content of Ephedra sinica. J. Nat. Med., 64, 63-69 (2010).
6) Kondo N, Mikage M, Idaka K. Medico-botanical studies of Ephedra plants from the Himalayan region. Part III. The causative factors of variation of alkaloid content in herbal stems. J. Nat. Med., 53, 194-200 (1999).

7) Zhu S, Kitani Y, Komatsu K. Exploration of Ephedra resource in Mongolia: from field investigation to molecular identification and chemical evaluation. J. Trad. Med., 29, 35-40 (2012).

8) Hong H, Chen HB, Yang DH, Shang MY, Wang X, Cai SQ, Mikage M. Comparison of contents of five ephedrine alkaloids in three official origins of Ephedra Herb in China by high-performance liquid chromatography. J. Nat. Med., 65, 623-628 (2011).

9) Matsumoto M, Hirayama M, Ohtomi N, Ohno T, Nomura Y, Iida O, Sugimura K, Kawahara N, Tsuchida T, Mikage M. Influence of genetic factors on the ephedrine alkaloid composition ratio of Ephedra plants. J. Nat. Med., 69, 63-67 (2015).

10) Hiyama H, Ozawa A, Kumazawa H, Takeda O. Stabilization of ephedrine alkaloid content in Ephedra sinica by selective breeding and stolon propagation. Biol. Pharm. Bull., 40, 43-48 (2017).

11) Japan Meteorological Agency. Past meteorological data download; 2019. Database: figs [Internet]. : 〈https://www.data.jma.go.jp/gmd/ risk/obsdl/index.php), accessed 31 October, 2020.

12) Kajimura K, Iwamoto Y, Yamasaki K, Sakagami Y, Yokoyama H, Yoneda K. Variation of growth and contents of ephedrine type alkaloids in Ephedra distachya. J. Nat. Med., 48, 122-125 (1994). 